\title{
Spectrophotometric analysis of crown discoloration induced by MTA- and ZnOE-based sealers
}

\author{
Konstantinos IOANNIDIS', Ilias MISTAKIDIS², Panagiotis BELTES ${ }^{3}$, Vassilis KARAGIANNIS ${ }^{4}$
}

1- DDS, MSc, Specialist in Endodontics, London, United Kingdom.

2- DDS, MSc, Department of Basic Dental Sciences, School of Dentistry, Aristotle University of Thessaloniki, Greece.

3- Professor, Department of Endodontology, School of Dentistry, Aristotle University of Thessaloniki, Greece.

4- PhD, Mathematician, Department of Mathematics, Aristotle University of Thessaloniki, Greece.

Corresponding address: loannidis Konstantinos - 300 Vauxhall Bridge Road, SW1V1AA - London - UK - Phone: 00447414979270 - e-mail: pabloioannidis@yahoo.com

Received: March 23, 2012 - Modification: January 3, 2013 - Accepted: January 30, 2013

\section{ABSTRACT}

\begin{abstract}
Crown discoloration can be induced by root canal sealer remnants following root canal treatment. Objective: The aim of this study was to evaluate chromatic alterations in human tooth crowns induced by a Mineral Trioxide Aggregate-based sealer (MTA Fillapex ${ }^{\circledR}$ ) and a commonly used ZnOE-based sealer (Roth-811). The tested null hypothesis was that the application of the materials did not induce clinically perceptible crown discoloration (Ho: CIE color difference $\Delta \mathrm{E}<3.7$ ). Material and Methods: Forty five fully developed, intact, mandibular third molars were sectioned $1 \mathrm{~mm}$ below the cemento-enamel junction. The pulp chambers were chemomechanically debrided via the cervical access. The specimens were randomly assigned into three groups Group 1: MTA Fillapex, Group 2: Roth 811, Group 3: Negative control (unfilled) and immersed in individually marked vials containing distilled water up to the cervix $\left(37 \pm 1^{\circ} \mathrm{C}\right)$. The spectral reflectance lines were recorded by utilizing a UV-VIS spectrophotometer equipped with integration sphere in the visual spectrum at baseline, 1 week, 1 and 3 months after material placement. Data were transformed into values of the CIE L*a*b* color system and the corresponding $\Delta E$ values were calculated. Statistical analysis was performed using two-way mixed ANOVA models, at $p=0.05$ level of significance. Results: A statistically significant increase in a* and b* chromatic parameters of the MTA Fillapex Group was measured. However, $\Delta \mathrm{E}$ values did not exceed the human eye perceptibility threshold (set at $\Delta \mathrm{E}<3.7$ ) during the experimental period $(\Delta \mathrm{Et} 3=2.88$ ). In Roth-811 Group, a statistically significant decrease in L* and a statistically significant increase in $a^{*}$ and $b^{*}$ chromatic parameters was measured, during all observation periods. Resultant $\Delta \mathrm{E}$ values exceeded the human eye perceptibility threshold after 1 week $(\Delta \mathrm{Et} 1=5.65)$. Conclusions: Application of MTA Fillapex in tooth crowns resulted in minimal color alterations, while Roth 811 induced severe discoloration, in vitro. It could be suggested that, in terms of aesthetics, the use of MTA Fillapex appears to be favorable.
\end{abstract}

Key words: CIE color system. Discoloration. MTA Fillapex. Root canal sealer. Roth 811. Spectrophometry.

\section{INTRODUCTION}

Poor aesthetic appearance of endodontically treated teeth remains a challenging issue in clinical dentistry even nowadays. Interestingly, it has been reported that poor aesthetic appearance of a treated tooth significantly affects the patients' quality of life ${ }^{10}$.

A major etiological factor for the occurrence of local intrinsic staining, especially in the cervical and middle third of the crown, is the presence of root canal filling materials in contact with the coronal dentin of the pulp chamber ${ }^{16,17}$. In the long-term, core materials and sealers interact with dentin. Any 
change to the optical and chromatic properties of the dentinal structure is likely to cause an alteration in the outward appearance of the crown caused by its light transmitting and reflecting properties ${ }^{16,17}$.

Apart from thorough pulp chamber debridement, a reduction of the coronal aspect of the root canal filling below the clinical cervix is required for the prevention of sealer-induced crown discoloration in the anterior aesthetic zone. However, sealer remnants cannot be always thoroughly removed from the pulp chamber and sometimes are present due to iatrogenic inadequate manipulations.

Despite the improvement of physicochemical, biomechanical and biological properties of endodontic sealers, the appearance of coronal discoloration is still evident in daily practice. Several laboratory studies have shown that some categories of sealers including ZnOE and epoxybased sealers are capable of inducing moderate to severe crown discoloration ${ }^{17,24,25,29}$. In endodontics, ZnOE sealers are used in clinical practice for many decades and are regarded as a gold standard in several laboratory and clinical studies. These materials are regarded to be clinically satisfactory, providing reasonable seal in the root canal system ${ }^{13}$. However, after setting reaction, the formation of a weak porous mass leads to dissolution in contact with tissue fluids ${ }^{8}$. Due to gradual hydrolysis, the release of eugenol leads to long-lasting cytotoxicity and additional potential for sensitization ${ }^{6}$.

Recently, Mineral Trioxide Aggregate (MTA) Fillapex ${ }^{\circledR}$ was introduced as a new generation MTAbased sealer. The main concept for the development of MTA-based sealers is the exploitation of the physical and biological properties of MTA such as bioactivity ${ }^{12}$, biocompatibility ${ }^{21}$ and hard tissue conductivity 22

A developing amount of research data is becoming available upon MTA Fillapex, with regard to its physical and biological properties ${ }^{2,13,14,23,26}$. Considering the increasing demands for aesthetics, biomaterials should be chromatically stable, present optical properties similar to dental structures and not exert staining effects to hard dental tissues ${ }^{17}$. Recent reports showed that both White and Gray MTA formulations are capable of inducing tooth discoloration ${ }^{4,5}$. From that perceptive, it is mandatory for every new MTA-based material to be tested in terms of aesthetic and color objectives. Currently, there are no available studies investigating the potential of MTA Fillapex to induce color alterations to dental tissues.

The aim of this study was to evaluate the chromatic alterations in human tooth crowns induced by MTA Fillapex and Roth 811 root canal sealer. The null-hypothesis $\left(\mathrm{H}_{0}\right)$ to be tested was that the application of the materials did not induce clinically perceptible crown discoloration $\left(\mathrm{H}_{\mathrm{o}}\right.$ : $\mathrm{CIE}$ color difference $\Delta \mathrm{E}<3.7)^{19}$.

\section{MATERIAL AND METHODS}

\section{Preparation of teeth}

Forty five freshly extracted, fully developed, impacted and semi-impacted mandibular third molars free of cracks, fractures, caries, abrasions and discoloration due to systemic intrinsic causes were collected, according to the guidelines of good clinical practice (Department of Oral and Maxillofacial Surgery, School of Dentistry, Ethical Committee, Aristotle University of Thessaloniki, Greece).

Soft tissue was removed and the teeth were sectioned in the coronal third of the root complex, $1 \mathrm{~mm}$ below the buccal cemento-enamel junction. Sectioning was performed with the aid of a lowspeed diamond-edge rotary saw microtome (Leica RM2255, Leica, Wetzlar, Germany). Access cavity preparation was not performed. Pulps were extirpated with a dental spoon and the internal axial walls of the pulp chambers were chemomechanically debrided with Hedstrom files (No. $\# 60-80)$ and $10 \mathrm{ml}$ of sodium hypochlorite (2.5\% w/w), through the apical access. Gentle reaming of the internal axial walls was performed through all directions. Between every file sequence, the pulp chamber was irrigated with $2 \mathrm{ml}$ of sodium hypochlorite $(2.5 \% \mathrm{w} / \mathrm{w}$ ) (3 file changes $\times 2 \mathrm{ml}=6$ $\mathrm{ml})$. A final rinse with $4 \mathrm{ml}$ of sodium hypochlorite $(2.5 \% \mathrm{w} / \mathrm{w})$ was performed at the end of the debridement. The pulp chambers of the specimens were finally washed with $5 \mathrm{ml}$ sterile saline to remove sodium hypochlorite remnants.

At the beginning of the experimental period, all crowns were transferred and stored in individually marked polyethlylene tubes containing distilled water up to the cervix of the crown in an incubator at $37 \pm 1^{\circ} \mathrm{C}$. The teeth $(\mathrm{N}=45)$ were randomly assigned in one experimental $(n=15)$, one positive $(n=15)$ and one negative control group $(n=15)$. The materials to be evaluated were MTA Fillapex (Angelus, Londrina, Brazil) (Group 1) and a ZnOE based sealer, Roth 811 (Roth's International, Chicago, IL) (Group 2) (Figure 1). The sealers were mixed and prepared according to the manufacturers' instructions and were placed into the pulp chambers via the cervical access. A finger plugger was used to coat the internal axial walls with the sealers. The apical access was sealed with a thin layer of glassionomer cement (Ketac Cem Aplicap, 3M, Espe, Germany) in order to address microleakage and sealers solubility. Negative controls (Group 3) were only instrumented and remained unfilled. 
Measurement of crown chromatic alterations

A double-beam UV-Vis spectrophotometer equipped with integrating sphere was used (UV2401PC, Shimadzu Corporation, Kyoto, Japan) and a standardized mounting system was developed, utilizing a previously validated experimental model $^{17}$. Standard D65 illumination was chosen (Commission Internationale de L'Eclairage 1978), as it corresponds approximately to the spectrum of midday daylight in Western/Northern Europe. The spectrophotometer was linked to a computer, which recorded the spectral reflectance curves of the crowns, in visual spectrum (380-780 nm).

The obtained spectral curves in visual spectrum (380-780 nm) were transformed into $L^{*}, a^{*}$ and $b^{*}$ values of the perceptually uniform CIE L*, a*, b* color space using a specialized computer software (Color Analysis UV-2401PC). L* values describe lightness, which range from black (0) to white (100), while a* values represent red (+80a*) to green $\left(-80 a^{*}\right)$, and $b^{*}$ values represent yellow $\left(+80 b^{*}\right)$ to blue $\left(-80 b^{*}\right)$ color variations. Total color differences $(\Delta \mathrm{E})$ were calculated according to the equation:

$\Delta \mathrm{E}=\left[\left(\mathrm{L}_{\mathrm{i}}-\mathrm{L}_{0} *\right)^{2}+\left(\mathrm{a}_{\mathrm{i}}-\mathrm{a}_{0} *\right)^{2}+\left(\mathrm{b}_{\mathrm{i}}-\mathrm{b}_{0} *\right)^{2}\right]^{1 / 2}$

The proposed acceptance for color matching adopted in this study was at $3.7 \Delta \mathrm{E}$ units (perceptibility threshold), beyond which the differences are clinically perceptible ${ }^{19}$. In dental science, when $\Delta \mathrm{E}$ values are less than 1 unit, then color match occurs and any color differences cannot be identified by independent observers in vitro ${ }^{27}$. However, color determination in clinical dentistry may become complicated by adjacent anatomic structures and lighting conditions. As a result, the proposed acceptance for color matching in dentistry has been set to 3.7 units (perceptibility threshold), beyond which differences are clinically visible ${ }^{19}$.

A standardized mounting system was developed for the customization and the reproducibility of the crown's position. The cylindrical inner frame (diameter $=2 \mathrm{~cm}$, inner height $=0.2 \mathrm{~cm}$ ) of the black bakelite sample assembly that the spectrophotometer was equipped with, was filled with black, non-polychromatic, thermo-plasticized silicone. The lingual surface of each crown was fixed within the silicone mass during its setting phase in order to construct individualized specimen carriers. The same individualized, silicone carrier for each specimen was used for measurements in all time intervals. The specimens were positioned in the in the circular opening of the aperture mask of the integrating sphere with the aid of an aligning system in order to ensure the reproducibility of the measured surface.

The dimensions of the polychromatic beam that illuminated the sample were $7 \times 7 \mathrm{~mm}$; thus the majority of the cervix and the crown surface was exposed and measured. The color appearance of the buccal surfaces of the crowns was measured in order to simulate their clinical appearance. Measurements were carried out by the same operator (I.M.) and at ambient temperature of $23 \pm 1^{\circ} \mathrm{C}$.

The spectrophotometer was calibrated at each time interval using $\mathrm{BaSO}_{4}$ reference. Measurements were performed prior to the placement of the materials (baseline: $t_{0}$ ) and consecutively 1 week $\left(t_{1}\right), 1$ month $\left(t_{2}\right)$ and 3 months $\left(t_{3}\right)$ after sealer placement. All measurements were repeated twice and averaged. If the total color difference $(\Delta E)$ between 2 measurements taken in a row exceeded the threshold of $1 \mathrm{DE}$ unit, new measurements were obtained.

At the end of the $3^{\text {rd }}$ month, three crowns of the experimental groups were randomly selected and longitudinally sectioned vertically to the middle of their mesio-distal dimension, on a bucco-lingual aspect, with the aid of a low-speed diamondedge rotary saw microtome (Leica RM2255, Leica, Wetzlar, Germany). Digital images of the sectioned crown specimens were taken, in day-light conditions (Macro-Lense 100 mm, Canon EOS 1000D, Tokyo, Japan).

\begin{tabular}{|c|c|c|c|c|}
\hline Group & $\begin{array}{c}\text { Sample } \\
\text { size (n) }\end{array}$ & $\begin{array}{c}\text { Materials under } \\
\text { study }\end{array}$ & Manufacturer & Composition \\
\hline Group 1 & 15 & MTA Fillapex & $\begin{array}{c}\text { Angelus, Londrina, } \\
\text { Brazil }\end{array}$ & $\begin{array}{r}\text { resins (salicylate, diluting, natural), } \\
\text { bismuth trioxide, nanoparticulated silica, } \\
\text { mineral trioxide } \\
\text { aggregate, pigments }\end{array}$ \\
\hline $\begin{array}{c}\text { Group 2 } \\
\text { (positive } \\
\text { control) }\end{array}$ & 15 & Roth 811 & $\begin{array}{c}\text { Roth's International, } \\
\text { Chicago, IL }\end{array}$ & $\begin{array}{c}\text { Powder: Zinc oxide, bismouth bicarbonate, } \\
\text { barium sulfate, dehydrated tetraboric sodium } \\
\text { Liquid: Chemically pure eugenol }\end{array}$ \\
\hline $\begin{array}{c}\text { Group 3 } \\
\text { (negative } \\
\text { control) }\end{array}$ & 15 & Unfilled & & \\
\hline
\end{tabular}

Figure 1- Study groups 1-3 


\section{Statistical analysis}

Sample size was calculated after power analysis based on the results of a pilot study, according to the equation $n=2\left(z_{1-a / 2}+z_{\beta}\right)^{2} \sigma^{2}\{1+(m-1) \rho\} /$ $m \Delta^{2}$ with $\rho=0.5, \mathrm{~m}=4$ (repeated measurements), $\sigma^{2}=4, \Delta=2.2$, alpha=0.05, power $=0.8$ (the chosen value of $\Delta$ was greater than the observed one in order to be close to the 3.7 threshold value and analogously done for the $\left.\sigma^{2}\right)^{17}$. Accordingly the sample size to each group was calculated near to $\mathrm{n}=12$ specimens per group. In case of drop out during the experimental period, 3 specimens were added in each group $(n=15)$.

Two-way ANOVA with repeated measurements was used for data analysis of the values of CIE $L^{*}, a^{*}, b^{*}$ chromatic parameters and the total color differences $(\Delta \mathrm{E})$. The significant effects and interactions of the experimental factors were investigated with pairwise between-group and within-group comparisons, which were conducted with Bonferroni's method. The overall analysis was performed with SPSS software (version 16.0, SPSS Inc., Chicago, IIl, USA). The level of statistical significance was set at $p<0.05$.

\section{RESULTS}

Tables 1 and 2 present the mean $L^{*}, a^{*}, b^{*}$ and $\Delta \mathrm{E}$ values of all groups, in all time intervals respectively. With regard to $L^{*}, a^{*}$ and $b^{*}$ parameters, MTA Fillapex caused a statistically significant increase in $\mathrm{a} *\left(\Delta \mathrm{a} * \mathrm{t}_{\mathrm{o}}-\mathrm{t}_{3}=1.043, \mathrm{p}<0.001\right)$ and $b^{*}\left(\Delta b^{*} t_{0}-t_{3}=2.292, p<0.001\right)$ values. $L^{*}$ values remained stable. However, resultant $\Delta \mathrm{E}$ values indicate that MTA Fillapex induced overall color changes below the human perceptibility threshold, ranging between 2.12-2.88 units. Roth 811 induced severe color changes exceeding the perceptibility threshold from the $1^{\text {st }}$ week of evaluation ( $\Delta \mathrm{E}=5.65)$. A statistically significant increase in a* $\left(\Delta a * t_{0}-t_{3}=-3,335, p<0.001\right)$ and b* $\left(\Delta b^{*} t_{0}-t_{3}=-4,014, p<0.001\right)$ values was observed, while $L^{*}$ values statistically decreased $\left(\Delta \mathrm{L}^{*} \mathrm{t}_{\mathrm{o}}-\mathrm{t}_{3}=4,926, \mathrm{p}<0.001\right)$. In Group 3, $\mathrm{L} *$, $\mathrm{a} *, \mathrm{~b} *$ values remained stable in all time intervals.

The macroscopic examination of the sectioned specimens of Group 1 (MTA Fillapex) showed stability of sealer color within its mass and absence of dentinal staining (Figure 2). On the contrary, in Group 2 (Roth 811) the set sealer displayed a granular, grayish appearance. Dark orange

Table 1- CIE L*, $a^{*}, b^{*}$ mean standard deviation values of Groups 1-3, in all experimental periods

\begin{tabular}{|c|c|c|c|c|c|}
\hline & & baseline & $1^{\text {st }}$ week & $1^{\text {st }}$ month & $3^{\text {rd }}$ month \\
\hline & \multicolumn{3}{|c|}{ (CIE) L* parameter } & & \\
\hline Groups & $\mathbf{n}$ & $\mathrm{L}_{0}$ & $\mathrm{~L}_{1}$ & $\mathrm{~L}_{2}$ & $\mathbf{L}_{3}$ \\
\hline MTA Fillapex & 15 & $84.95(3.14)$ & $85.36(2.54)$ & $84.35(3.10)$ & $84.91(2.61)$ \\
\hline Roth 811 & 15 & $84.21(1.98)$ & $81.89(1.18)$ & $79.38(1.82)$ & $79.28(1.71)$ \\
\hline \multirow[t]{3}{*}{ Negative control } & 15 & $84.55(2.22)$ & $84.77(2.26)$ & $84.48(2.34)$ & $84.55(2.17)$ \\
\hline & & baseline & $1^{\text {st }}$ week & $1^{\text {st }}$ month & $3^{\text {rd }}$ month \\
\hline & \multicolumn{3}{|c|}{ (CIE) a* parameter } & & \\
\hline Groups & $\mathbf{n}$ & $a_{0}$ & $a_{1}$ & $a_{2}$ & $a_{3}$ \\
\hline MTA Fillapex & 15 & $0.15(0.68)$ & $0.57(0.67)$ & $0.77(0.68)$ & $1.19(0.65)$ \\
\hline Roth 811 & 15 & $0.01(0.79)$ & $2.03(0.82)$ & $2.71(1.00)$ & $3.34(1.05)$ \\
\hline \multirow[t]{3}{*}{ Negative control } & 15 & $0.42(0.74)$ & $0.39(0.66)$ & $0.38(0.73)$ & $0.41(0.73)$ \\
\hline & & baseline & $1^{\text {st }}$ week & $1^{\text {st }}$ month & $3^{\text {rd }}$ month \\
\hline & \multicolumn{3}{|c|}{ (CIE) b* parameter } & & \\
\hline Groups & $\mathbf{n}$ & $b_{0}$ & $b_{1}$ & $b_{2}$ & $b_{3}$ \\
\hline MTA Fillapex & 15 & $21.60(2.82)$ & 23.36(2.21) & $22.64(2.74)$ & $23.89(2.35)$ \\
\hline Roth 811 & 15 & $21.26(2.33)$ & $25.67(2.36)$ & $24.76(2.72)$ & $25.28(2.89)$ \\
\hline Negative control & 15 & $20.62(3.11)$ & $20.33(2.89)$ & $20.49(2.94)$ & $20.57(3.07)$ \\
\hline
\end{tabular}

Within-group comparisons: a statistically significant differences when compared to $t_{0}$, $b$ when compared to $t_{1}$, c when compared to $t_{2}, d$ when compared to $t_{3}$

Between-group comparisons: a statistically significant differences when compared to Fillapex, B when compared to Roth 811 
Table 2- Mean standard deviation $\Delta \mathrm{E}$ values of Groups 1-3 in all experimental periods

\begin{tabular}{ccccc}
\hline & & $1^{\text {st }}$ week & $1^{\text {st }}$ month & 3 $^{\text {rd }}$ month \\
Groups & $\mathbf{n}$ & $\left(\Delta \mathrm{E}_{1}\right)$ & $\left(\Delta \mathrm{E}_{2}\right)$ & $\mathbf{( \Delta \mathrm { E } _ { 3 } )}$ \\
\hline MTA Fillapex & 15 & $2.13(1.18)$ & $2.12(0.72)$ & $2.88(1.49)$ \\
Roth 811 & 15 & $5.65(2.34)$ & $6.70(2.68)$ & $7.37(3.02)$ \\
Negative control & 15 & $0.51(0.37)$ & $0.61(0.30)$ & $0.51(0.20)$ \\
\hline
\end{tabular}

Within-group comparisons: a statistically significant differences when compared to $\Delta \mathrm{E}_{1}$, b when compared to $\Delta \mathrm{E}_{2}$, $\mathrm{c}$ when compared to $\Delta \mathrm{E}_{3}$

Between-group comparisons: A statistically significant differences when compared to MTA Fillapex, B when compared to Roth 811

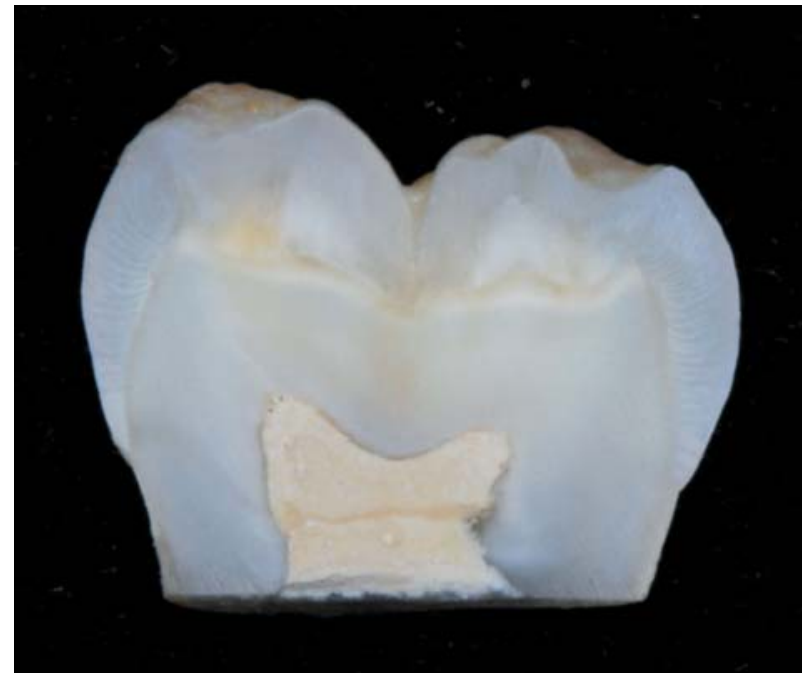

Figure 2- Appearance of Group 1 (MTA Fillapex) after 3 months. Color stability of MTA Fillapex sealer within its mass and absence of dentinal staining

dentinal staining accompanied by varying leaching within tubules was evident, in areas where sealer remnants were in contact with the axial walls of the pulp chamber (Figure 3).

\section{DISCUSSION}

MTA Fillapex is a newly developed MTA-based root canal sealer. According to the manufacturer, its composition after mixture is basically MTA particles incorporated in a matrix of salicylate resin, natural resin, bismuth and silica. Other proposed MTAbased sealers consist of MTA with other additives, including HEMA, TEGDMA resins (IC-MTA) and watersoluble polymers (ProRoot-Endo sealer) ${ }^{7,11}$. Recent data reported that MTA Fillapex presents acceptable physical properties, antibacterial properties and intracanal resistance to dislodgement similar to $\mathrm{AH}$ Plus $^{2,23}$. The sealer presents increased cytotoxicity during the setting reaction which decreases over time ${ }^{26}$. After setting, the sealer is capable of retaining alkaline $\mathrm{pH}$ levels due to calcium release, and presents bioactivity stimulating hydroxyapatite crystal nucleation in human osteoblast-like cell

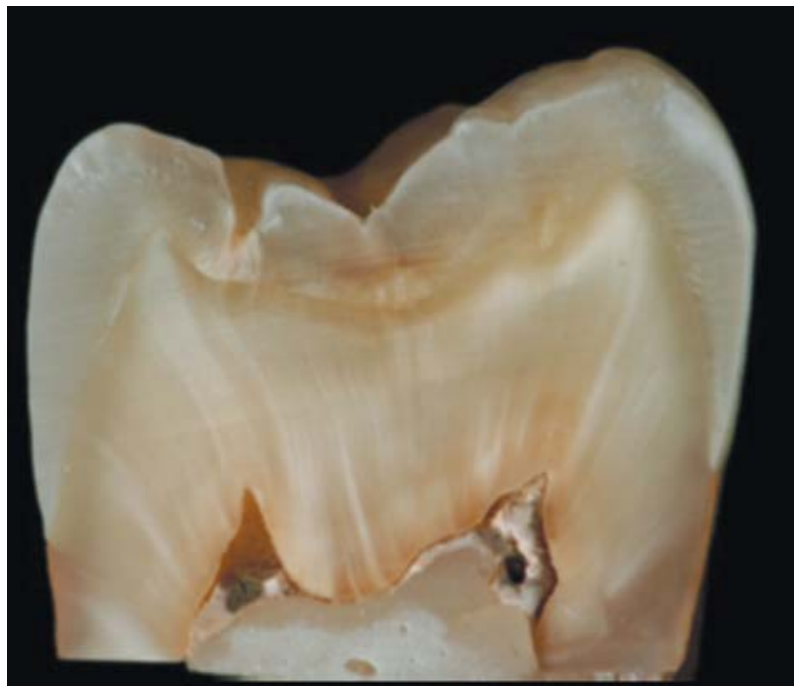

Figure 3- Appearance of Group 2 (Roth 811) after 3 months. Granular, grayish appearance of Roth 811 sealer and dark orange dentinal staining accompanied by varying leaching within tubules

cultures $^{26}$ and mineralization in connective tissue ${ }^{14}$.

The rational for organizing this research study was the increasing publication of case reports, in which the discoloring effect of MTA has been reported. Both Gray and White MTA repair materials were capable of inducing coronal discoloration, when applied in cases of root and furcal perforation, pulpotomy and pulp revascularization procedures ${ }^{4,5}$. In addition, two recent laboratory studies confirmed the previous clinical observations with the aid of instrumental colorimetry $^{1}$ and visual spectrophotometry ${ }^{18}$. It has been proposed that metal oxides, such as iron and manganese, could be responsible for the discoloring effects of Gray MTA ${ }^{18}$. Moreover, recent data have shown that the discoloring effects of White MTA have been attributed to its progressive mass darkening due the presence of reduced black crystals of bismuth atoms under light conditions ${ }^{28}$.

This laboratory study is the first to report tooth color alterations induced by MTA Fillapex, utilizing the methodology of visual spectrophotometry. Within the limitations of this experimental study, in 
acceptance of the null hypothesis, MTA Fillapex did not induce clinically perceptible crown discoloration. In rejection of the null hypothesis, Roth 811 induced fast and severe discoloration and exceeded the perceptibility threshold 1 week after sealer placement.

The results of this study indicated that the tested sealer had minimum potential to induce color alterations in human teeth in vitro, since the measured color alterations did not exceed the threshold of perceptibility set $(\Delta E<3.7)$. The descriptive analysis of the CIE L*, a*, b* chromatic parameters showed a statistically significant color change towards red and yellow after three months, while lightness was not affected. The macroscopic findings confirmed that the sealer did not present color changes within its mass, maintaining its yellowish color throughout the experimental period. Dentinal staining was not evident as well. The increase of CIE $a *$ and $b^{*}$ values of the crowns from the first week of investigation may be attributed to alterations in specimens' optical properties due to the physical presence of the sealer in the internal dentinal surface.

Roth 811 sealer induced fast and severe discoloration, which was clinically perceptible 1 week following sealer placement. The chromogenic potential of ZnOE sealers has been attributed to the unstable chemical bond between $\mathrm{ZnO}$ and eugenol. Even after the end of the setting reaction, eugenol release leads to self-oxidation and becomes darker with time. The results of this study are in complete accordance with results of previous studies $17,25,29$.

Several methods have been proposed for the evaluation or measurement of sealer-induced discoloration, including visual technique and computer analysis of digital photos ${ }^{24,25,29}$. Inherent objectivity and standardization difficulties may be improved by the use of a spectrophotometer ${ }^{15}$. This methodology has been reported as accurate and reliable in dentistry for quantitative tooth color measurements ${ }^{17,20}$. A major advantage of visual spectrophotometry is that tooth color measurement is based on the measurement of spectral reflectance, which describes the total reflection of a sample in visual spectrum ${ }^{17}$.

Random errors in this study were minimized by strict control of the environmental factors along with multiple measurements and averaging. One of the main limitations of instrumental color measurement, however, is posed by systematic errors that can be attributed to variations in instrument geometrical design, metamerism and calibration techniques ${ }^{27}$.

The comparison of absolute tristimulus values found in that study with other studies is discouraged. Evaluation and comparison of total color differences (expressed by $\Delta E$ ), however, is well documented and considered as safe since differential measurement is highly reproducible between instruments ${ }^{9}$. The results of this study are comparable to those of a previous study which was conducted utilizing the same methodology and under the same experimental conditions. After 3 months, non-perceptible total color differences $(\Delta \mathrm{E})$ of MTA Fillapex were similar to the $\Delta \mathrm{E}$ values of Guttaflow (Roeko, Coltene, Whaledent Ltd., Germany) and Epiphany SE (Pentron Clinical Technologies, Wallingford, CT), indicating that they likewise exert minimal chromogenic effects ${ }^{17}$.

The results of this study do not directly represent the in vivo tooth discoloration potential of root canal sealers in good clinical practice. In this laboratory study, the investigation of the discoloration potential of root canal sealers was based on the generation of a "worst case scenario" by leaving a significant amount of sealer in direct contact with the axial dentinal walls and several anatomical features of the pulp chamber ${ }^{17}$. However, the knowledge of the magnitude of the sealers' chromogenic potential indicates that thorough cleaning measures are essential to prevent discoloration postoperatively.

Regardless of the minimal staining effects of new generation root canal sealers including MTA Fillapex, the clinician should always ensure thorough removal of sealer remnants. Apart from basic properties, such as biocompatibility and good sealing ability, it could be suggested that the chromogenic potential of sealers may also play an important role in selecting proper root canal filling materials.

\section{CONCLUSION}

Within the limitations of this experimental study, MTA Fillapex did not induce clinically perceptible crown discoloration. Roth 811 induced fast and severe discoloration and exceeded the perceptibility threshold 1 week after sealer placement. Although the incorporated MTA has proved chromogenic potential, MTA Fillapex posed minimal risk for potential staining effects. It could be suggested that, in terms of aesthetics, the use of MTA Fillapex appears to be favorable.

\section{REFERENCES}

1- Akbari M, Rouhani A, Samiee S, Jafarzadeh H. Effect of dentin bonding agent on the prevention of tooth discoloration produced by mineral trioxide aggregate. Int ] Dent. 2012 [cited 2013 Feb 15];2012:563203. Available from: http://dx.doi. org/10.1155/2012/563203.

2- Assmann E, Scarparo RK, Bottcher DE, Grecca FS. Dentin bond strength of two mineral trioxide aggregate-based and one epoxy resin-based sealers. J Endod. 2012;38:219-21.

3- Barnett F, Trope M, Rooney J, Tronstad L. In vivo sealing ability of calcium hydroxide-containing root canal sealers. Endod Dent Traumatol. 1989;5:23-6.

4- Belobrov I, Parashos P. Treatment of tooth discoloration after the use of white mineral trioxide aggregate. J Endod. 2011;37:101720. 
5- Bortoluzzi EA, Araújo GS, Guerreiro Tanomaru JM, TanomaruFilho M. Marginal gingival discoloration by Gray MTA: a case report. J Endod. 2007;33:325-7.

6- Brodin P. Neurotoxic and analgesic effects of root canal cements and pulp-protecting dental materials. Endod Dent Traumatol. 1988;4:1-11.

7- Camilleri J, Mallia B. Evaluation of the dimensional changes of mineral trioxide aggregate sealer. Int Endod J. 2011;44:416-24. 8- Carvalho-Júnior JR, Guimarães LF, Correr-Sobrinho L, Pécora JD, Sousa-Neto MD. Evaluation of solubility, disintegration, and dimensional alterations of a glass ionomer root canal sealer. Braz Dent J. 2003;14:114-8.

9- Douglas RD. Precision of in vivo colorimetric assessments of teeth. J Prosthet Dent. 1997; 77:464-70.

10- Dugas NN, Lawrence HP, Teplitsky P, Friedman S. Quality of life and satisfaction outcomes of endodontic treatment. J Endod. 2002;28:819-27.

11- Gandolfi MG, Taddei P, Siboni F, Modena E, Ciapetti G, Prati C. Development of the foremost light-curable calcium-silicate MTA cement as root-end in oral surgery. Chemical-physical properties, bioactivity and biological behavior. Dent Mater. 2011;27:e134-57. 12- Gandolfi MG, Taddei P, Tinti A, Prati C. Apatite-forming ability (bioactivity) of ProRoot MTA. Int Endod J. 2010;43:917-29. 13- Gomes-Filho JE, Moreira JV, Watanabe S, Lodi CS, Cintra LT, Dezan E Jr, et al. Sealability of MTA and calcium hydroxide containing sealers. J Appl Oral Sci. 2012;20:347-51.

14- Gomes-Filho JE, Watanabe S, Lodi CS, Cintra LT, Nery MJ, Otoboni JA Filho, et al. Rat tissue reaction to MTA FILLAPEX ${ }^{\circledR}$. Dent Traumatol. 2012;28:452-6.

15- Guan YH, Lath DL, Lilley TH, Willmot DR, Marlow I, Brook $\mathrm{AH}$. The measurement of tooth whiteness by image analysis and spectrophotometry: a comparison. J Oral Rehabil. 2005;32:7-15. 16- Ioannidis K, Beltes $P$, Lambrianidis T, Kapagiannidis D, Karagiannis V. Crown discoloration induced by endodontic sealers. Spectrophotometric measurement of Commission International de I'Eclairage's L*, a*, b* chromatic parameters. Oper Dent. 2013. In press.

17- Ioannidis K, Beltes P, Lambrianidis T, Kapagiannidis D, Karagiannis $\mathrm{V}$. Validation and spectrophotometric analysis of crown discoloration induced by root canal sealers. Clin Oral Inv. 2013 [cited Feb 27]. Available from: http://dx.doi.org/10.1007/ s00784-012-0850-x.
18- Ioannidis K, Mistakidis I, Beltes P, Karagiannis V. Spectrophotometric analysis of coronal discolouration induced by grey and white MTA. Int Endod J. 2013;46:137-44.

19- Johnston WM, Kao EC. Assessment of appearance match by visual observation and clinical colorimetry. J Dent Res. $1989 ; 68: 819-22$.

20- Karamouzos A, Papadopoulos MA, Kolokithas G, Athanasiou $\mathrm{AE}$. Precision of in vivo spectrophotometric colour evaluation of natural teeth. J Oral Rehabil. 2007;34:613-21.

21- Mitchell PJ, Pitt Ford TR, Torabinejad M, McDonald F. Osteoblast biocompatibility of mineral trioxide aggregate. Biomater. 1999;20:167-73.

22- Moretton TR, Brown CE Jr, Legan JJ, Kafrawy AH. Tissue reactions after subcutaneous and intraosseous implantation of mineral trioxide aggregate and ethoxybenzoic acid cement. J Biomed Mater Res. 2000;52:528-33.

23- Morgental RD, Vier-Pelisser FV, Oliveira SD, Antunes FC, Cogo DM, Kopper PM. Antibacterial activity of two MTA-based root canal sealers. Int Endod J. 2011;44:1128-33.

24- Parsons JR, Walton RE, Ricks-Williamson L. In vitro longitudinal assessment of coronal discoloration from endodontic sealers. J Endod. 2001;27:699-702.

25- Partovi M, Al-Havvaz AH, Soleimani B. In vitro computer analysis of crown discolouration from commonly used endodontic sealers. Aust Endod J. 2006;32:116-9.

26- Salles LP, Gomes-Cornélio AL, Guimarães FC, Herrera BS, Bao SN, Rossa-Junior C, et al. Mineral trioxide aggregate-based endodontic sealer stimulates hydroxyapatite nucleation in human osteoblast-like cell culture. J Endod. 2012;38:971-6.

27- Seghi RR, Hewlett ER, Kim J. Visual and instrumental colorimetric assessments of small color differences on translucent dental porcelain. J Dent Res. 1989;68:1760-4.

28- Vallés $M$, Mercadé $M$, Duran-Sindreu F, Bourdelande JL, Roig $M$. Color stability of white mineral trioxide aggregate. Clin Oral Inv. 2013 [cited Feb 27]. Available from: http://dx.doi.org/10.1007/ s00784-012-0794-1.

29- Van der Burgdt TP, Mullaney TP, Plasschaert AJM. Tooth discoloration induced by endodontic sealers. Oral Surg Oral Med Oral Pathol. 1986;61:84-9. 